\title{
Efficacy of Hypertonic Saline and Normal Saline in the Treatment of Chronic Sinusitis
}

\author{
Ramabhadraiah Anil Kumar, Borlingegowda Viswanatha, Nisha Krishna, \\ Niveditha Jayanna, Disha Ramesh Shetty \\ Department of ENT, Bangalore Medical College \& Research Institute, Bangalore, India \\ Email: drbviswanath@yahoo.co.in
}

Received March 18, 2013; revised April 23, 2013; accepted May 3, 2013

Copyright (C) 2013 Ramabhadraiah Anil Kumar et al. This is an open access article distributed under the Creative Commons Attribution License, which permits unrestricted use, distribution, and reproduction in any medium, provided the original work is properly cited.

\begin{abstract}
Introduction: Chronic sinusitis affects all age groups and is a cause for significant morbidity. Recent realization that noninfectious inflammatory causes can predispose to infectious sinusitis has evoked renewed interest in developing and documenting efficacious ancillary therapies that could supplement antibiotic use. Hypertonic saline solution has been shown to increase mucociliary clearance and ciliary beat frequency. Objectives: A double blinded randomized comparative study was undertaken to evaluate the effect of hypertonic saline (3.5\%) nasal drops and normal saline $(0.9 \%)$ nasal drops, to assess the tolerance of hypertonic saline nasal drops and to know if hypertonic nasal drops improve the "quality of life" in patients with chronic sinusitis. Methods: Fifty patients diagnosed as chronic sinusitis in the age group of 18 - 45 years were randomized into two groups; Group A was treated with normal saline and Group B with 3.5\% hypertonic saline for a period of 4 weeks. Pre and Post treatment x-rays of the paranasal sinuses (Water's view) were graded and radiological scores were given accordingly. The symptoms were evaluated before and after treatment using visual analogue score. Patients were queried about tolerance to the nasal solution and scores were given. Conclusion: Hypertonic saline nasal solution is more efficacious than normal saline solution in the treatment of patients with chronic sinusitis. Hypertonic saline nasal solution was well tolerated and it improved quality of life in these patients.
\end{abstract}

Keywords: Chronic Sinusitis; Hypertonic Saline; Normal Saline

\section{Introduction}

Paranasal sinus disease is a common illness seen in the general population. It is one of the leading causes for absenteeism from work, frequent revisits to the doctor and also a cause for significant expenditure of money on over-the-counter medications.

The common modalities of treatment for chronic sinusitis include the use of antibiotics, decongestants, mucolytics and steroids. Long term use of these medicines can have a detrimental effect both locally and systemically. Nasal mucociliary function becomes impaired in most patients who have chronic upper respiratory tract infections. There has been recent realization that noninfectious causes can predispose to infectious sinusitis. This has led many doctors to advocate the use of ancillary treatment for chronic sinusitis.

Nasal irrigation aids in the clearance of secretions, debris and intranasal crusts. This is also important in the postoperative period to reduce the risk of adhesions and to promote osteomeatal patency. Use of normal saline and hypertonic saline for nasal irrigation is an inexpensive technique that can be used alone or along with other interventional modalities for nose and paranasal sinus diseases.

Improvement in mucociliary clearance is well documented, with the use of normal saline as well as hypertonic saline. However, controversy exists regarding the beneficial effects of hypertonic saline over normal saline in reducing symptoms of chronic sinusitis.

This study was designed to compare the efficacy of hypertonic saline nasal drops over that of normal saline nasal drops in the treatment of chronic sinusitis. As per the null hypothesis it was assumed that there is no difference between the two groups and finally the results were compared which showed a statistically significant result.

\section{Materials and Methods}

The present prospective randomized comparative study was conducted at Victoria Hospital, Department of 
Otorhinolaryngology, Bangalore Medical College and Research Institute, Bangalore, Karnataka, India from July 2009 to August 2011.The research project was approved by the Ethics Committee of the Institution under the protocol Good Clinical Practices.

During this period, fifty patients who were diagnosed with chronic sinusitis in the age group of 18 - 45 years were selected. They were randomized into two groups. Group A included twenty-five cases treated with normal saline (solution A), ten drops three times a day in both nostrils for a period of 4 weeks and the remaining twenty-five cases in Group B were treated with 3.5\% hypertonic saline (solution B), ten drops three times a day in both nostrils for the same period.

Patients who had been treated with antibiotics, $\beta_{2}$ agonists, topical steroids and systemic steroids were included in the study, but the treatment was stopped one month prior to the beginning of the study. Diagnosis was confirmed by x-ray paranasal sinuses (Water's view). Patients with fever who were treated with antibiotics and steroids during the study and those with any known anatomical defect or mucocele that obstructs the sinuses were excluded from the study.

Detailed history was taken and clinical examination carried out in selected cases. The diagnosis of chronic sinusitis was made with two major criteria; nasal discharge, postnasal drip and one minor criteria, headache. The diagnosis was confirmed by x-ray of paranasal sinus (Water's view). Post treatment x-ray of the paranasal sinuses (Water's view) was taken at the end of 4 to 6 weeks and compared with the pretreatment x-ray. The pre and post treatment $\mathrm{x}$-rays were graded according to Berg et al. [1] (1981) (Table 1), by a consultant who was blinded about the mode of treatment for each side of the sinus, and change in grading was recorded. Radiological scores were given accordingly as mentioned below.

The patients were informed about the mode of treatment and asked to report every week for a period of one month to assess symptoms. Normal saline which is commercially available as $0.9 \%$ Sodium chloride solution was used as solution A. Hypertonic solution of 3.5\% Sodium chloride was prepared by dissolving $3.5 \mathrm{~g}$ of sodium chloride in $100 \mathrm{ml}$ of double distilled water and was used as solution B. The solution was dispensed in $100 \mathrm{ml}$ sterile bottles.

In our study a concentration of 3.5\% saline solution was chosen because its concentration is similar to that of sea water, moreover it is considered harmless and is better tolerated by patients, even children. Nasal solution in the form of drops is easy to dispense, cost effective, simple to prepare and does not require any special device for delivery; also aerosol deposition in lower airways can be prevented.

Patients were randomly selected and either solution A or solution B was given as nasal drops. They were ad- vised to instill ten drops intranasally three times a day. The drops were instilled fast upward in a sitting or standing position with the head pulled back to allow secretions to flow downwards from the nose without the patient breathing them in. They were immediately removed from the nose in order to minimize the salty taste and burning sensation that may occur.

The symptoms were evaluated using visual analogue score. The score ranged from 0 - $10(0=$ none and $10=$ most severe) for nasal congestion, headache, facial pain, sense of smell, nasal discharge; helping in overall symptomatic assessment. This was done once a week during the treatment period and once at the end of the treatment. Quality of life was assessed using Visual Analogue Score (VAS) [2]. Patients were also queried about tolerance for the nasal solution, which was assessed using scores $1=$ No burning sensation; 2 = Mild burning sensation; 3 = Moderate burning sensation and $4=$ Severe burning sensation.

Data was analyzed from findings recorded in the predesigned proforma. Chi-square test was used to find the independence of groups at 5\% level of significance. Unpaired " $t$ " test was used to find significant difference between the groups.

\section{Results and Analysis}

The following observations were made in forty two patients who completed the treatment schedule proposed in the study. Eight patients (16\%) defaulted from the study group of fifty patients.

Patients in group B had significant improvement in nasal congestion by the end of fourth week when compared to group A (Table 2).

Patients reported relief of headache in both groups but comparison between the two groups showed significant improvement in group B (Table 3) by the end of fourth week.

We observed that facial pain subsided in both the groups by the end of second or third week of treatment and the difference was found to be statistically insignificant between the two groups (Table 4).

Loss of smell was reported by eight patients in group $A$ and in eleven patients in group $B$ and in both the groups it improved with treatment. However, while comparing the 2 groups (Table 5), the difference was found to be statistically insignificant.

Patients reported clear nasal secretions and reduction in the quantity of secretions by the end of fourth week in both the groups. But when compared with group A, group B showed significant improvement by the end of fourth week (Table 6).

In our study it was observed that both groups showed improvement in their overall symptoms but, group B showed significant improvement when compared to 
Table 1. Radiological grading of X-ray paranasal sinuses according to Berg et al. [1].

\begin{tabular}{ccc}
\hline Grade & Radiological Finding & Radiological Score \\
\hline I & No mucosal hypertrophy & 1 \\
II & Mucosal thickening of $<0.5$ cm but no fluid level & 2 \\
III & Mucosal thickness $>0.5$ cm but no fluid level & 3 \\
IV & Attenuating tissue or fluid occupying sinuses or fluid level & 4 \\
\hline
\end{tabular}

Table 2. Treatment analysis of nasal congestion (VAS) in group A and group B.

\begin{tabular}{ccccc}
\hline VAS & Group & No. of Patients & Mean \pm Standard Deviation & T \\
\hline \multirow{2}{*}{ 1st - 2nd week } & A & 21 & $1.48 \pm 0.98$ & 2.11 \\
& B & 21 & $2.15 \pm 0.93$ & $\mathrm{p}=0.035 \mathrm{sig}$ \\
1st - 3rd week & A & 21 & $2.86 \pm 1.20$ & 3.68 \\
& B & 21 & $4.10 \pm 0.85$ & $\mathrm{p}=0.001 \mathrm{vhs}$ \\
1st - 4th week & A & 21 & $5.43 \pm 1.12$ & 2.582 \\
& B & 21 & $6.25 \pm 0.79$ & $\mathrm{p}=0.01 \mathrm{hs}$ \\
\hline
\end{tabular}

"sig—significant, vhs—very highly significant, hs—highly significant.

Table 3. Treatment analysis of headache (VAS) in group A and group B.

\begin{tabular}{ccccc}
\hline VAS & Group & No. of Patients & Mean \pm Standard Deviation & T \\
\hline \multirow{2}{*}{ 1st - 2nd week } & A & 21 & $1.48 \pm 0.81$ & 2.178 \\
& B & 21 & $2.05 \pm 0.69$ & $\mathrm{p}=0.029$ sig \\
1st - 3rd week & A & 21 & $3.19 \pm 1.44$ & 2.631 \\
& B & 21 & $4.45 \pm 1.00$ & $\mathrm{p}=0.009 \mathrm{hs}$ \\
1st - 4th week & A & 21 & $5.10 \pm 1.26$ & 2.738 \\
& B & 21 & $6.20 \pm 0.95$ & $\mathrm{p}=0.006 \mathrm{hs}$ \\
\hline
\end{tabular}

"sig—significant, vhs—very highly significant, hs—highly significant. Difference between the two groups at the end of 4 weeks is highly significant ( $\mathrm{p}=$ 0.006).

Table 4. Treatment analysis of facial pain (VAS) in group A and group B.

\begin{tabular}{ccccc}
\hline VAS & Group & No. of Patients & Mean \pm Standard Deviation & T \\
\hline \multirow{2}{*}{ 1st - 2nd week } & A & 3 & $0.24 \pm 0.62$ & 1.733 \\
& B & 1 & $0.00 \pm 0.00$ & $\mathrm{p}=0.083 \mathrm{n} \mathrm{sig}$ \\
1st - 3rd week & A & 3 & $0.38 \pm 1.02$ & 1.733 \\
& B & 1 & $0.00 \pm 0.00$ & $\mathrm{p}=0.029 \mathrm{n} \mathrm{sig}$ \\
1st - 4th week & A & 3 & $0.38 \pm 1.02$ & 1.690 \\
& B & 1 & $0.00 \pm 0.00$ & $\mathrm{p}=0.029 \mathrm{n} \mathrm{sig}$ \\
\hline
\end{tabular}

Table 5. Treatment analysis of smell (VAS) in group A and group B.

\begin{tabular}{|c|c|c|c|c|}
\hline VAS & Group & No. of Patients & Mean \pm Standard Deviation & $\mathbf{T}$ \\
\hline \multirow{2}{*}{ 1st - 2nd week } & A & 8 & $2.33 \pm 1.22$ & \multirow{2}{*}{$\begin{array}{c}1.021 \\
\mathrm{p}=0.307 \mathrm{n} \mathrm{sig}\end{array}$} \\
\hline & $\mathrm{B}$ & 11 & $2.55 \pm 0.69$ & \\
\hline \multirow{2}{*}{ 1st - 3rd week } & A & 8 & $4.89 \pm 2.03$ & \multirow{2}{*}{$\begin{array}{c}0.936 \\
\mathrm{p}=0.350 \mathrm{n} \text { sig }\end{array}$} \\
\hline & B & 11 & $5.36 \pm 1.21$ & \\
\hline \multirow{2}{*}{ 1st - 4th week } & A & 8 & $6.22 \pm 1.56$ & \multirow{2}{*}{$\begin{array}{c}0.492 \\
p=0.623 \text { n sig }\end{array}$} \\
\hline & $\mathrm{B}$ & 11 & $6.09 \pm 0.83$ & \\
\hline
\end{tabular}


group A. X-rays were graded and given scores accordingly. There was no statistical significance in distribution of cases between the two groups. In group B, highly significant downgrading in radiological score was noticed (from $5.67 \pm 1.32$ to $3.62 \pm 1.43$ ); whereas in group $\mathrm{A}$ there was no such improvement (from $5.38 \pm 1.43$ to 4.71 $\pm 1.42)(p=0.001)$ (Table 7).

In group A $85.7 \%$ patients did not complain of any burning sensation. Mild burning sensation was reported by $14.3 \%$ in group $\mathrm{A}$ and $57.1 \%$ in group $\mathrm{B}$; it was highly significant in group B when compared to group A. Moderate burning sensation was reported by $19 \%$ of patients in group B. None of the patients in both the groups reported severe burning sensation (Table 8).

\section{Discussion}

It is well known that the usual treatment modalities in chronic sinusitis include antibiotics, decongestants, mucolytics and steroids. Long term use of these drugs can have a detrimental effect, both locally and systemically. Hypertonic saline nasal irrigation was formally identi- fied as an adjunctive care for sinusitis in 1990s [3-6] though it was advocated since Vedic times. In Hatha Yoga, Jala-neti is described as a nasal cleansing technique for sinonasal diseases [7]. Our study showed that hypertonic saline nasal solutions used to reduce symptoms of chronic sinusitis was more efficacious than normal saline nasal solution. In various studies different concentrations of hypertonic saline solutions have been used. We used $3.5 \%$ saline solution because it is about the concentration of sea water and is considered harmless and better tolerated by the patients, even children [6]. The mechanism of action is unclear. It has been hypothesized that it improves mucociliary function [8-10], decreases mucosal edema and inflammatory mediators [11], and mechanically clears inspissated mucus [12,13]. In addition, hypertonic saline is said to have a mild vasoconstrictive effect [14] and antibacterial property [15]. Hypererosmolarity of the airway fluids causes an increase in $\mathrm{Ca}^{2+}$ release from intercellular stores and increase in $\mathrm{Ca}^{2+}$ may stimulate the ciliary beat frequency, possibly by regulating the use or availability of adeno-

Table 6. Treatment analysis of nasal secretion (VAS) in group A and group B.

\begin{tabular}{ccccc}
\hline VAS & Group & No. of Patients & Mean \pm Standard Deviation & T \\
\hline \multirow{2}{*}{ 1st - 2nd week } & A & 21 & $1.57 \pm 0.87$ & 3.074 \\
& B & 21 & $2.45 \pm 0.89$ & $\mathrm{p}=0.002 \mathrm{hs}$ \\
1st - 3rd week & A & 21 & $3.24 \pm 1.55$ & 3.590 \\
& B & 21 & $4.65 \pm 0.93$ & $\mathrm{p}=0.001 \mathrm{v} \mathrm{hs}$ \\
1st - 4th week & A & 21 & $5.38 \pm 0.63$ & 3.210 \\
& B & 21 & $6.40 \pm 0.88$ & $\mathrm{p}=0.001 \mathrm{v}$ hs \\
\hline
\end{tabular}

Table 7. Treatment analysis of overall symptomatic relief (VAS) in group A and group B.

\begin{tabular}{|c|c|c|c|c|}
\hline VAS & Group & No. of Patients & Mean \pm Standard Deviation & $\mathbf{T}$ \\
\hline \multirow{2}{*}{ 1st - 2nd week } & A & 21 & $1.57 \pm 0.87$ & \multirow{2}{*}{$\mathrm{p}=0.002$ hs } \\
\hline & B & 21 & $2.45 \pm 0.89$ & \\
\hline \multirow{2}{*}{ 1st - 3rd week } & A & 21 & $3.24 \pm 1.55$ & \multirow{2}{*}{$\begin{array}{l}3.594 \\
p=0.001 \mathrm{v} h s\end{array}$} \\
\hline & B & 21 & $4.65 \pm 0.93$ & \\
\hline \multirow{2}{*}{ 1st - 4th week } & A & 21 & $5.38 \pm 0.73$ & \multirow{2}{*}{$\begin{array}{c}2.912 \\
p=0.003 h s\end{array}$} \\
\hline & $\mathrm{B}$ & 21 & $6.40 \pm 0.88$ & \\
\hline
\end{tabular}

Table 8. Tolerance to the nasal solution in group $A$ and group $B$.

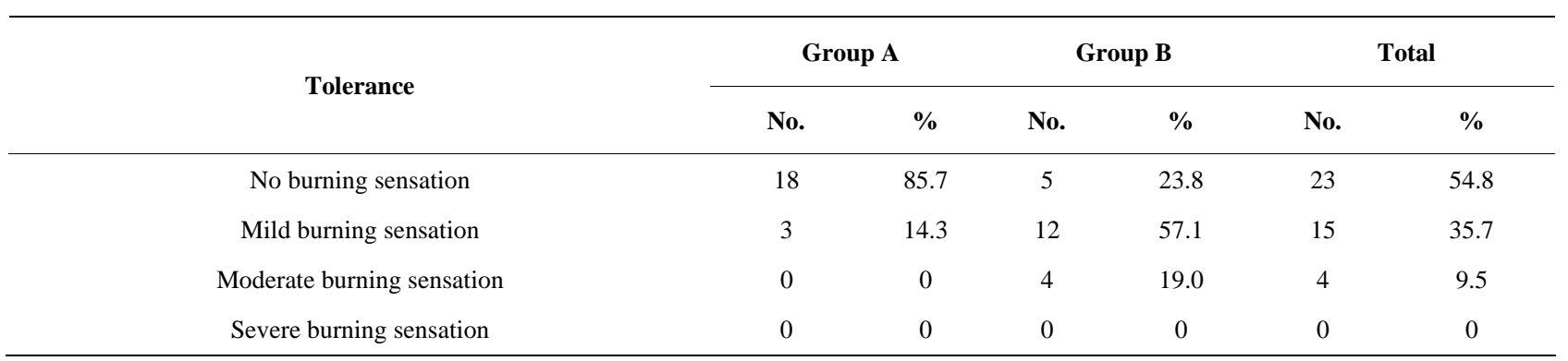


sine triphosphate by ciliary axoneme. Study of the pulmonary epithelial barrier showed that after instillation of hyperosmolar sea water, there is a rapid influx of water from the plasma into the bronchoalveolar space. Osmotic equilibrium was reached within 3 minutes and there was no injury to the epithelial or endothelial barriers of the lung [16]. Intrinsic ciliary beat frequency and ultrastructure are not inherently impaired in chronic sinusitis, because impaired ciliary function caused by chronic sinusitis reverses to normal after removal and cleansing the mucosa of infected mucous and other material [15]. It appears that respiratory ciliated cells have a functional reserve that permits them to autoregulate their mechanical output in response to changing respiratory mucus viscosity [17]. The dynamic viscoelastic properties of nasal mucosa determined by oscillary rheometry has revealed significant improvement in elasticity after repeated antral lavages in chronic sinusitis [18].

In our study of fifty patients $12 \%$ failed to report for follow up and $4 \%$ complained of burning sensation in the nose due to hypertonic saline nasal solution and were excluded from the study group. In a similar study, David Shoseyov [6] reported discontinuation of treatment due to burning sensation in the nose and throat in $8.82 \%$ of cases in the hypertonic saline group and $2.94 \%$ of the cases in the normal saline group. In contrast, none of the other similar studies reported burning sensation as a reason for noncompliance [19-21].

In our study almost all patients had nasal congestion, nasal secretions and headache. Few patients complained of loss of smell and facial pain. Each symptom was scored by Visual Analog Scale (VAS) and compared with the mean from the end of first and fourth week. A significant improvement in nasal congestion was observed in both the groups but it was found to be more pronounced in group $\mathrm{B}$.

In group $\mathrm{A}$, the mean difference between first week and fourth week was $5.43 \pm 1.12$ whereas that of group B was $6.25 \pm 0.79(p=0.01)$. These results are in sharp contrast with that of Lance T. Tomooka [19] who reported a net change in nasal congestion scores before and after treatment as $16.7(\mathrm{p}=0.0010)$ in patients treated with hypertonic nasal irrigation.

All forty two patients in the study group reported relief of headache with treatment but in group B relief from headache was much earlier and significant when compared to group A. In group A the mean difference between first week and fourth week was $5.10 \pm 1.26$ and that for group B was $6.20 \pm 0.95$ ( $=0.006)$. Only four patients in our study complained of facial pain, $14.28 \%$ from group A and $4.76 \%$ from group B. In group A, the mean difference between first week and fourth week was $0.38 \pm 1.02$ and that of group B was $0.00 \pm 0.00(\mathrm{p}=$ 0.029). This is in contrast to the observations made by
Tomooka [19], who reported a net change in symptom scores for headache and facial pain before and after treatment by $6.8(\mathrm{p}=0.058)$ in patients treated with hypertonic nasal irrigation. This may be explained by the fact that patients with other sinonasal diseases were included in Tomooka's [22] study and they used $150 \mathrm{ml}$ of $2 \%$ saline buffered with baking soda for nasal irrigation for 6 months. We used 10 drops of 3.5\% hypertonic saline that was instilled by a nasal dropper for 4 weeks and the study group consisted of patients with chronic sinusitis only where headache was one of the inclusion criteria.

We observed that sense of smell improved with treatment in both the groups and there was no significant difference between the groups at the end of the study. Loss of smell was reported by nine patients in group A and in eleven patients in group B. In group A the mean difference between the first week and fourth week was $6.22 \pm$ 1.56 and that of group B was $6.09 \pm 0.83(p=0.623)$.

Improvement in nasal secretions scores was observed in both the groups in our study. However a significant improvement in group B was noted, as postnasal drip/ nasal secretions disappeared or became clear in most of the patients. In group A the mean difference between first week and fourth week was $5.38 \pm 0.63$ and that of group B was $6.40 \pm 0.88(\mathrm{p}=0.001)$.

Even though there was improvement; there was no significant difference between the groups treated with 3.5\% hypertonic and $0.9 \%$ normal saline nasal drops.

Overall symptomatic assessment in group A showed that mean difference between first week and fourth week scores was $5.38 \pm 0.73$ and that of group B was $6.40 \pm$ $0.88(\mathrm{p}=0.003)$.

Visual analog score (VAS) was used which is a global quality-of-life indicator unlike true utilities (which assess the desirability of health states versus an external metric).

Radiological analysis in group A showed that there were nine patients with right maxillary sinus haziness; eight with left sided haziness and four with bilateral haziness. In group B there were eleven patients with right maxillary sinus haziness; seven with left sided haziness and three had bilateral haziness. The analysis of pretreatment and post-treatment radiological scores revealed highly significant improvement in group B (from $5.67 \pm$ 1.32 to $3.62 \pm 1.43$ ) compared to group A (from $5.38 \pm$ 1.43 to $4.71 \pm 1.42)(p=0.001)$. Figure 1 shows radiological improvement in one of the patients.

Tolerance to $0.9 \%$ normal saline nasal solution used as solution A in group A and 3.5\% hypertonic saline nasal solution used as solution B in group B, showed moderate burning sensation in 19\% of patients in group B and none in group A. Mild burning sensation was reported in $14.3 \%$ and $57.1 \%$ in group A and B respectively. In group A $85.7 \%$ and in group B $23.8 \%$ did not complain 


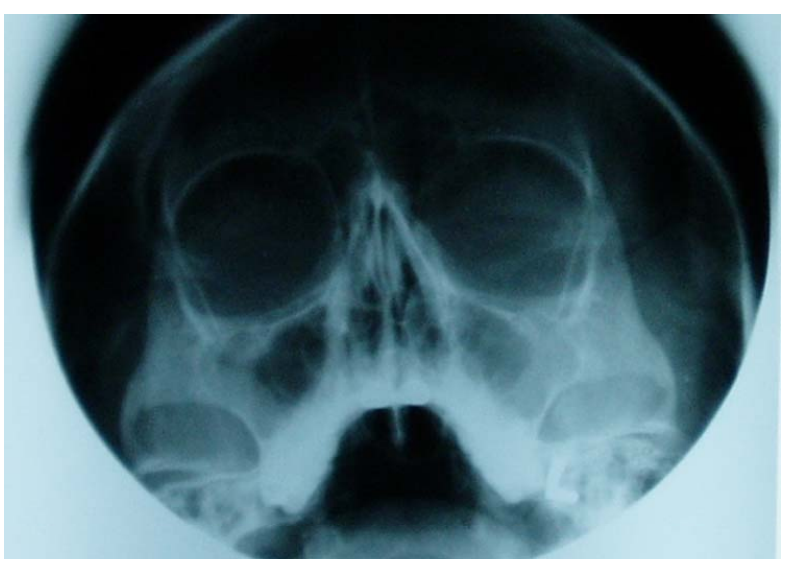

Pre-treatment

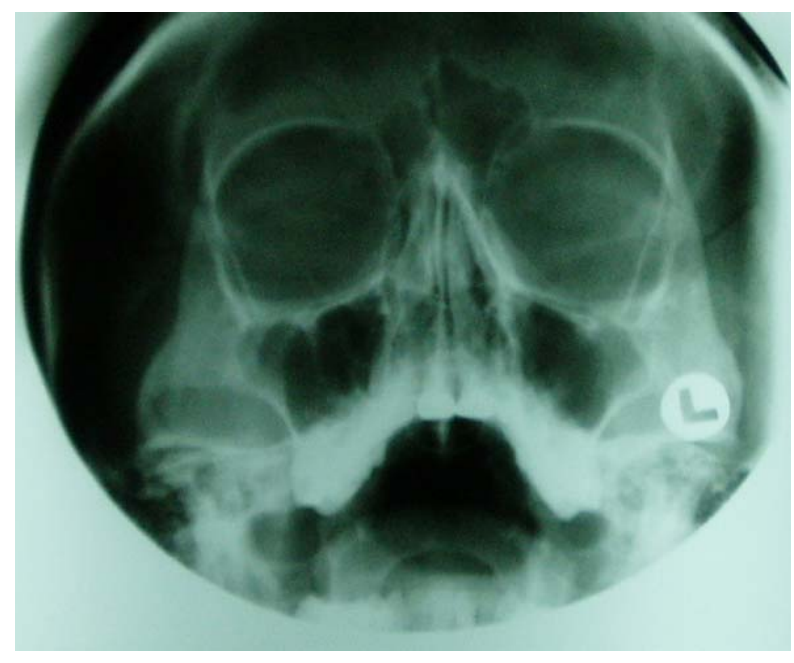

Post-treatment

Figure 1. Showing radiological improvement after treatment with hypertonic saline (3.5\%) nasal drops.

of burning sensation. None of the patients in both the groups reported severe burning sensation. Though burning sensation was reported by the patients with hypertonic saline solution, it was well tolerated.

\section{Conclusion}

The study showed that 3.5\% hypertonic saline nasal solution was more efficacious than $0.9 \%$ normal saline solution in the treatment of patients with chronic sinusitis. Hypertonic saline nasal solution was well tolerated by the patients and the treatment of patients with chronic sinusitis with $3.5 \%$ hypertonic saline nasal solution improved their quality of life.

\section{REFERENCES}

[1] O. Berg, H. Bergstedt, C. Carenfelt and M. G. Zind, “Discrimination of purulent from non-purulent maxillary sinusitis: Clinical and radiographic diagnosis," The Annals of Otology, Rhinology, and Laryngology, Vol. 90, No. 3,
1981, pp. 272-275.

[2] I. S. Mackay and V. J. Lund, "Surgical Management of Sinusitis: Scott-Brown's Otolaryngology,” 6th Edition, Butterworth Heinemann Publishers, Oxford, 1997.

[3] W. Wingrave, "The Nature of Discharges and Douches," The Lancet, Vol. 17, 1902, pp. 1373-1375.

[4] M. Grossan, "Irrigation of the Child's Nose," Clinical Pediatrics, Vol. 13, No. 3, 1974, pp. 229-231. doi:10.1177/000992287401300306

[5] R. S. Zeiger, "Prospects for Ancillary Treatment of Sinusitis in the 1990s," Journal of Allergy and Clinical Immunology, Vol. 90, No. 3, 1992, pp. 478-495. doi:10.1016/0091-6749(92)90173-Y

[6] D. Shoseyov, H. Bibi, P. Shai, N. Shoseyov, G. Shazberg and H. Hurvitz, "Treatment with Hypertonic Saline versus Normal Saline Nasal Wash of Pediatric Chronic Sinusitis," Journal of Allergy and Clinical Immunology, Vol. 101, No. 5, 1998, pp. 602-605. doi:10.1016/S0091-6749(98)70166-6

[7] "Instruction Manual and General Information on Yogic Saline Nasal Cleansing Technique. The Jala-neti Booklet," 2005.

http://www.yogaage.com/asanas/jala.pdf

[8] B. C. Band, A. L. Mukherjee and F. B. Bang, "Human Nasal Mucous Flow Rates,” The Johns Hopkins Medical Journal, Vol. 121, No. 1, 1967, pp. 38-48.

[9] M. Grossan, “A Device for Nasal Irrigation,” ANL, Vol. 3, 1976, pp. 65-70.

[10] Y. Majima, Y. Sakakura, T. Matsubara, S. Murai and Y. Miyoshi, "Mucociliary Clearance in Chronic Sinusitis: Related Human Nasal Clearance and in Vitro Bullfrog Palate Clearance,” Biorheology, Vol. 20, No. 2, 1983, pp. 251-262.

[11] J. W. Georgitis, "Nasal Hyperthermia and Simple Irrigation for Perennial Rhinitis," Chest, Vol. 106, No. 5, 1994, pp. 1487-1491. doi:10.1378/chest.106.5.1487

[12] M. J. Dulfano, K. Adler and O. Wooten, "Primary Properties of Sputum. IV. Effects of 100 Percent Humidity and Water Mist," American Review of Respiratory Disease, Vol. 107, No. 1, 1973, pp. 130-132.

[13] C. M. Rossman, R. M. Lee, J. B. Forrest and M. T. Newhouse, "Nasal Ciliary Ultrastructure and Function in Patients with Primary Ciliary Dyskinesia Compared with That in Normal Subjects and in Subjects with Various Respiratory Diseases,” American Review of Respiratory Disease, Vol. 129, No. 1, 1984, pp. 161-170.

[14] S. C. Manning, "Pediatric Sinusitis," Otolaryngologic Clinics of North America, Vol. 26, No. 4, 1993, pp. 623638.

[15] E. D. O. Mangete, D. West and C. D. Blankson, "Hypertonic Saline Solution for Wound Dressing (Letter),” The Lancet, Vol. 340, No. 8831, 1992, p. 1351. doi:10.1016/0140-6736(92)92533-L

[16] H. G. Folkesson, F. Kheradmand and M. A. Matthay, "The Effect of Salt Water on Alveolar Epithelial Barrier Function," American Journal of Respiratory and Critical Care Medicine, Vol. 150, No. 6, 1994, pp. 1555-1563. doi:10.1164/ajrccm.150.6.7952614 
[17] N. T. Johnson, M. Villalon, F. H. Royce, R. Hard, P. Verdugo, "Autoregulation of Beat Frequency in Respiratory Ciliated Cells. Demonstrated by Viscous Loading," American Review of Respiratory Disease, Vol. 144, No. 5, 1991, pp. 1091-1094. doi:10.1164/ajrccm/144.5.1091

[18] K. Hirata, "Dynamic Viscoelasticity of Nasal Mucus from Children with Chronic Sinusitis," Mie Medical Journal, Vol. 34, 1985, pp. 205-219.

[19] L. T. Tomooka, "Claire Murphy and Terence M Davidson. Clinical Study and Literature Review of Nasal Irrigation,” Laryngoscope, Vol. 110, No. 7, 2000, pp. 1189-1193. doi:10.1097/00005537-200007000-00023

[20] D. G. Heatley, K. E. McConnell, T. L. Kille and G. E.
Leverson, "Nasal Irrigation for the Alleviation of Sinonasal Symptoms," Otolaryngology-Head and Neck Surgery, Vol. 125, No. 1, 2001, pp. 44-48. doi:10.1067/mhn.2001.115909

[21] D. Rabago, A. Zgierska, M. Mundt, B. Barrett, J. Bobula and R. Maberry, "Efficacy of Daily Hypertonic Saline Nasal Irrigation among Patients with Sinusitis,” The Journal of Family Practice, Vol. 51, No. 12, 2002, pp. 10491055.

[22] M. Taccariello, A. Parikh, Y. Darby and G. Scadding, "Nasal Douching as a Valuable Adjunct in the Management of Chronic Rhinosinusitis,” Rhinology, Vol. 37, No. 1, 1999, pp. 29-32. 